\title{
Targeting angiogenesis in advanced non-small-cell lung cancer: are biomarkers needed?
}

\author{
G. Metro • M. Cenci • A. Baldi • L. Crinò
}

Published online: 13 April 2013

(C) Springer Science+Business Media New York 2013

\begin{abstract}
Tumor-related angiogenesis is a complex process resulting from a delicate balance between pro and antiangiogenic factors. Bevacizumab, a fully humanized monoclonal antibody against vascular endothelial growth factor (VEGF), was the first anti-angiogenic therapy to prove effective, in combination with chemotherapy, for treatment of several types of advanced cancer, non-small-cell lung cancer (NSCLC) included. However, as for other types of solid malignancy, no predictive biomarkers of increased sensitivity to bevacizumab or any other anti-angiogenic drugs could be identified in NSCLC, which has had a negative affect on the cost-effectiveness of the targeted therapy performed to block the VEGF pathway. In this review we discuss the most relevant findings from clinical and correlative studies investigating the use of antiangiogenic therapy for advanced NSCLC, focusing on the need to identify biomarkers for selection of patients suitable for anti-angiogenic therapy.
\end{abstract}

Keywords Angiogenesis · Bevacizumab · Non-small-cell lung cancer $\cdot$ VEGF $\cdot$ VEGFR-TKI

\section{Introduction}

First postulated in the 1970s by Judah Folkman and colleagues, angiogenesis, namely the development of new

\footnotetext{
G. Metro $(\varangle) \cdot$ M. Cenci $\cdot$ A. Baldi $\cdot$ L. Crinò $(\varangle)$

Division of Medical Oncology, Santa Maria della Misericordia

Hospital, Azienda Ospedaliera di Perugia, via Dottori, 1, 06156 Perugia, Italy

e-mail: giulio.metro@yahoo.com

e-mail: lucio.crino@ospedale.perugia.it

M. Cenci

e-mail: matteocenci77@yahoo.it

A. Baldi

e-mail: alice87.ab@libero.it
}

blood vessels from parent vessels, has emerged as a crucial regulator of growth and survival of human malignancies, including lung cancer $[1,2]$. Briefly, angiogenesis results from a balance between pro and antiangiogenic factors; in tumors the former strongly predominate [3•]. Among others, the dominant process regulating angiogenesis is the interaction between the vascular endothelial growth factor proteins (VEGFs), in particular VEGF-A (or VEGF), and their receptors (VEGFRs 1 to 3 ), located on the tumor vasculature (blood and/or lymphatic), which, by means of dimerization, induce the downstream signaling cascade leading to proliferation, migration, and permeability of endothelial cells. As a result of these processes, tumor growth and formation of metastases are promoted [2]. For this reason, blockade of the interaction between VEGF and VEGFRs has been proposed as a potential antitumor treatment. To achieve this, two main strategies have been investigated, either use of monoclonal antibodies against VEGF or use of molecules that inhibit the tyrosine-kinase activity of VEGFRs. In this review we briefly discuss the anti-angiogenic drugs that have been investigated for treatment of advanced lung cancer, particularly emphasizing the urgent need for biomarkers that could help predict sensitivity to anti-angiogenic treatment.

\section{Targeting angiogenesis in NSCLC}

\section{Bevacizumab}

Bevacizumab (Avastin), a recombinant humanized monoclonal antibody directed against the VEGF, is the only antiangiogenic agent approved by the US Food and Drug Administration (FDA) and the European Medicines Agency (EMA) for treatment of lung cancer. It should be prescribed in addition to carboplatin-paclitaxel (FDA) or platinumbased chemotherapy (EMA) for first-line treatment of 
advanced NSCLC patients with non-squamous histology [4, 5]. This was based on the results of two phase III studies, the E4599 and AVAiL trials, in which the combination of bevacizumab with platinum-based chemotherapy resulted in significantly superior progression-free survival (PFS) compared with platinum-based chemotherapy alone [6-8]. However, overall survival (OS) was significantly improved in the E4599 trial only $(\mathrm{HR}=0.79)$, which adopted carboplatin-paclitaxel as chemotherapy regimen in combination with bevacizumab [6]. Nevertheless, more recently, a metaanalysis of summarized data from four randomized phase II and/or phase III trials comparing first-line bevacizumab plus platinum-based chemotherapy versus platinum-based chemotherapy alone showed that bevacizumab-based treatment significantly prolongs PFS and OS, with estimated HRs of 0.72

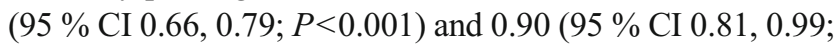
$P=0.03$ ), respectively [9॰]. These results reinforce the effect of bevacizumab in combination with platinum-based chemotherapy as first-line therapy for advanced non-squamous NSCLC, which is clearly acknowledged by major international guidelines, including those from the National Comprehensive Cancer Network (NCCN) and the European Society for Medical Oncology (ESMO) [10, 11].

However, despite having been consistently associated with a significant clinical benefit in advanced nonsquamous NSCLC, it cannot be neglected that the absolute increase in one-year survival obtained with bevacizumab plus platinum-based chemotherapy has been estimated to be as modest as $4 \%$ (from $51 \%$ to $55 \%$ ) [ $9 \cdot$ ]. Therefore, major attempts should be made to identify biomarkers of sensitivity that would aid selection of patients suitable for bevacizumab-based treatment. In fact, identifying the patients who may benefit most from such therapy would certainly prevent unresponsive individuals from being exposed to the low but significant increase in non-trivial severe adverse effects of bevacizumab, including, but not limited to, proteinuria, hypertension, and hemorrhage [9•]. Paradoxically, only clinical criteria for exclusion from bevacizumab treatment have yet been identified, which may be helpful for minimization of toxicity (i.e. pulmonary hemorrhage) rather than for prediction of sensitivity to treatment (Table 1) [12•]. Against this background, cost-utility analysis, on the basis of models which take into account survival, toxicity, and costs of treatment, has shown that use of bevacizumab in addition to carboplatin-paclitaxel (as used in E4599) is not likely to be cost-effective compared with platinum-based chemotherapy alone [13].

\section{Candidate biomarkers of sensitivity to bevacizumab}

Certainly, several factors challenge the identification of biomarkers of sensitivity to bevacizumab treatment in NSCLC, including the inability to perform serial biopsies in order to
Table 1 Clinical factors justifying exclusion from bevacizumab-based treatment for advanced NSCLC to minimize the risk of pulmonary hemorrhage

\begin{tabular}{ll}
\hline Clinical factor & Comment \\
\hline Predominantly squamous histology $^{\mathrm{a}}$ & $\begin{array}{c}\text { Exclusion criterion from pivotal } \\
\text { phase III trials }[6,7]\end{array}$ \\
History of grade $\geq 2$ hemoptysis & $\begin{array}{c}\text { Exclusion criterion from pivotal } \\
\text { phase III trials }[6,7]\end{array}$ \\
$\begin{array}{l}\text { Major blood vessel infiltration } \\
\text { and bronchial vessel infiltration, } \\
\text { encasement, and abutting }\end{array}$ & $\begin{array}{c}\text { Standardized radiological criteria } \\
\text { for defining infiltration have } \\
\text { not been established }\end{array}$ \\
\hline
\end{tabular}

${ }^{\mathrm{a}}$ Defined as $>50 \%$ of squamous cells in the sample used for histological diagnosis by a pathologist

${ }^{\mathrm{b}}$ Defined as bright red blood $\geq 2.5 \mathrm{~mL}$ per event

assess pre and post-treatment changes in tumor vasculature, the confounding effect that the chemotherapy regimen with which bevacizumab is combined has on treatment efficacy, and the compensating effect that alternative angiogenic signals other than VEGF exert on treatment outcome. Nevertheless, it is surprising that oncology research focusing on studies specifically aimed at identifying putative biomarkers of sensitivity to bevacizumab treatment has been initiated only recently (Table 2 ). In this context, baseline plasma levels of VEGF (pVEGF) have attracted much attention, on the basis of exploratory analysis of results from randomized trials showing that high levels (above median values) of short isoforms of pVEGF were predictive of improved PFS and/or OS while on bevacizumab treatment for patients with advanced breast, pancreatic and gastric cancers [14]. Unfortunately, these results, which seem to correlate bevacizumab efficacy with high VEGF dependency of sensitive tumors, could not be replicated in NSCLC patients treated in the AVAiL trial [14]. Similarly, no predictivity was shown for any major isoform of pVEGF in either the E4599 and AVAiL trials, although results from E4599 suggested that high pVEGF levels could be predictors of improved objective response rate (ORR) among patients treated with bevacizumab plus carboplatin and/or paclitaxel compared with those receiving carboplatin and/or paclitaxel alone (33.3 \% vs. $7.7 \%$, respectively, $P=0.01$ ) $[15,16]$. Several reasons could be proposed to explain these contradictory results. First, pVEGF may be of different predictive value depending on the tumor type for which it is evaluated. In addition, use of a single measurement time (i.e. at baseline) and difficulties in categorizing within cutoffs results from analysis of a continuous variable, for example pVEGF, certainly limit interpretation of results. Nevertheless, although not predictive, high levels of pVEGF were consistently shown to be predictors of worse survival of bevacizumab-untreated patients (irrespective of isoform), which suggests a biological effect of pVEGF in NSCLC 
Table 2 Main biomarker studies of bevacizumab efficacy in advanced NSCLC

\begin{tabular}{|c|c|c|c|c|}
\hline & $\begin{array}{l}\text { Biomarker } \\
\text { analysis }\end{array}$ & $\begin{array}{l}\text { Study } \\
\text { acronym }\end{array}$ & Study details & $\begin{array}{l}\text { Correlation with outcome for } \\
\text { bevacizumab-treated patients }\end{array}$ \\
\hline $\begin{array}{l}\text { Short VEGF } \\
\text { isoform [14] }\end{array}$ & Plasma & AVAiL & $\begin{array}{l}\text { Three groups, receiving placebo, } 7.5 \text {, or } \\
15 \mathrm{mg} / \mathrm{kg} \text { bevacizumab, each } \\
\text { combined with cisplatin-gemcitabine }\end{array}$ & $\begin{array}{l}\text { High VEGF levels: trend toward } \\
\text { improved PFS with bevacizumab } \\
15 \mathrm{mg} / \mathrm{kg}\end{array}$ \\
\hline \multirow[t]{3}{*}{$\begin{array}{l}\text { VEGF, all major } \\
\text { isoforms [15-18] }\end{array}$} & \multirow[t]{3}{*}{ Plasma } & E4599 & $\begin{array}{l}\text { Two groups, receiving either bevacizumab } \\
15 \mathrm{mg} / \mathrm{kg} \text { or placebo, each combined } \\
\text { with carboplatin-paclitaxel }\end{array}$ & High VEGF levels: improved ORR \\
\hline & & AVAiL & $\begin{array}{l}\text { Three groups, receiving placebo, } 7.5 \text {, or } \\
15 \mathrm{mg} / \mathrm{kg} \text { bevacizumab, each combined } \\
\text { with cisplatin-gemcitabine }\end{array}$ & None \\
\hline & & ABIGAIL & $\begin{array}{l}\text { Two groups, receiving either bevacizumab } \\
15 \mathrm{mg} / \mathrm{kg} \text { or } 7.5 \mathrm{mg} / \mathrm{kg} \text { both combined } \\
\text { with platinum-based chemotherapy (carboplatin- } \\
\text { paclitaxel or cisplatin-gemcitabine) }\end{array}$ & $\begin{array}{l}\text { High VEGF levels: reduced } \\
\text { PFS and OS }\end{array}$ \\
\hline $\begin{array}{l}\text { SNPs of selected } \\
\text { genes [19] }\end{array}$ & $\begin{array}{l}\text { Serum DNA of } \\
\text { VEGF, VEGFR-1, } \\
\text { and VEGFR-2 }\end{array}$ & ABIGAIL & $\begin{array}{l}\text { Two groups, receiving either bevacizumab } \\
15 \mathrm{mg} / \mathrm{kg} \text { or } 7.5 \mathrm{mg} / \mathrm{kg} \text { both combined } \\
\text { with platinum-based chemotherapy (carboplatin-- } \\
\text { paclitaxel or cisplatin-gemcitabine) }\end{array}$ & None \\
\hline Hypertension [26] & - & E4599 & $\begin{array}{l}\text { Two groups, receiving either bevacizumab } \\
15 \mathrm{mg} / \mathrm{kg} \text { or placebo, each combined with } \\
\text { carboplatin-paclitaxel }\end{array}$ & $\begin{array}{l}\text { High blood pressure: improved } \\
\text { PFS and OS }\end{array}$ \\
\hline
\end{tabular}

ORR, objective response rate; OS, overall survival; PFS, progression-free survival; SNP, single nucleotide polymorphism

$[14,16]$. Recently, the prognostic significance of pVEGF was also confirmed for bevacizumab treated patients [17, 18]. ABIGAIL was a randomized phase II study in which patients with advanced non-squamous NSCLC were allocated to receive one of two different doses of bevacizumab (either 7.5 or $15 \mathrm{mg} / \mathrm{kg}$ every three weeks) in combination with platinum-based chemotherapy (either carboplatin-paclitaxel or carboplatin-gemcitabine) selected according to the investigator's choice, the primary end-point being correlation between primary candidate plasma biomarkers and ORR [17]. Although the primary end-point was not met, because none of the biomarkers assessed at baseline, which included VEGF, VEGFR-1 and 2, ICAM, bFGF, E-selectin, and PLGF, correlated with response to treatment, high levels of pVEGF were reported to correlate with a worse outcome than low pVEGF levels, both in terms of PFS (6.1 vs. 7.4 months, respectively, $\mathrm{HR}=1.57 ; P=0.002)$ and $\mathrm{OS}(11.1 \mathrm{vs.}$ 19.8 months, respectively, $\mathrm{HR}=1.57 ; P=0.0042)[17,18]$. Nevertheless, because of the presence of bevacizumab in both types of treatment, this study could not differentiate between predictive versus prognostic value of pVEGF.

Although the utility of pVEGF as a predictive biomarker remains to be clarified, there is also increasing interest in the effect of VEGF genotype and VEGF single nucelotide polymorphism (SNPs). In this regard, the ABIGAIL study reported on the correlation between prespecified SNPs from three genes, VEGFA, VEGFR-1, and VEGFR-2, and clinical outcome [19]. The SNPs analyzed were selected on the basis of their potential predictive value previously observed not only in NSCLC, but also in breast and pancreatic cancer
[20-22]. Interestingly, three SNPs in VEGFA were associated with significantly increased best ORR, whereas one SNP in VEGFR-1 correlated with a statistically increased risk of progression or death [19]. However, statistical significance was lost after adjusting for multiple testing for all four SNPs.

In bevacizumab studies of NSCLC, tissue biomarkers have been less extensively studied than plasma biomarkers. VEGF expression may be an appealing biomarker in this setting, because its overexpression is almost invariably associated with poor prognosis [23]. In this regard, recent results from breast cancer studies suggest that patients with VEGF gene amplification may not benefit from bevacizumab, which is consistent with the hypothesis that this amplification "overwhelms" the ability of bevacizumab to successfully block VEGF [24].

Clinical criteria are sometimes helpful in suggesting benefit from targeted agents. For instance, the development of cutaneous rash has been suggested as a means of identifying patients who benefit from treatment with the anti-EGFR monoclonal antibody cetuximab [25]. Hypertension, a common side effect of antiangiogenic therapy, has also been proposed as a potential predictive biomarker of sensitivity of NSCLC patients to bevacizumab [26]. An exploratory analysis from E4599 revealed that among bevacizumabtreated patients those who experienced high blood pressure (HBP) at the end of cycle one had an OS of 15.9 months versus 11.5 months for patients without HBP. Similarly, bevacizumab treatment was associated with PFS of 7 . 0 months among HBP patients compared with 5.5 months 
for patients not experiencing HBP [26]. Conversely, no clinically significant differences between OS and PFS were observed among HBP and low-blood-pressure patients in the control group. Interestingly, the relationship between the development of hypertension and the activity of bevacizumab has been described for other solid malignancies also [27]. However, at this time, whether hypertension is a biomarker of bevacizumab activity remains to be determined in a prospective study.

VEGFR-tyrosine kinase inhibitors (TKIs) and other anti-angiogenic agents

The VEGFR-TKIs drugs include a plethora of small molecules whose mechanism of anti-tumor activity is based on inhibition of multiple receptor tyrosine kinases, including blockade of VEGFR(s). Among these drugs, vandetanib, cediranib, sorafenib, and motesanib have been tested in randomized clinical trials of chemonaïve or platinumpretreated advanced NSCLC patients with the common study design of comparing standard chemotherapy with the same chemotherapy regimen given in combination with the VEGFR-TKI [28-35]. Unfortunately, addition of a VEGFRTKI to chemotherapy was not found to improve overall survival. Nevertheless, some clinical activity was observed in the VEGFR-TKI plus chemotherapy group, given the significant improvement of ORR and/or PFS in most cases [29, 32-35]. Against this background, there is uncertainty about how to proceed with the clinical development of VEGFR-TKIs for treatment of advanced NSCLC; more importantly, questions of which patients should be treated with a VEGFR-TKI and how they should be treated, are of crucial importance.

There may be several reasons for the lack of success of VEGFR-TKIs in NSCLC. First, angiogenesis may not be the predominant mechanism of antitumor activity for most of the VEGFR-TKIs, as suggested by their capacity to inhibit multiple signaling pathways. For instance, because of its antagonist effect on raf-kinases, sorafenib may work best in KRAS-mutant NSCLCs [36]. Second, the lack of synergy of the VEGFR-TKI and chemotherapy combination is consistent with the preclinical observation that VEGFRTKIs may induce G1 cell cycle arrest in the tumor, thus potentially interfering with the cycle-dependent toxicity of concomitant chemotherapy $[37,38]$. That is why pharmacodynamic separation achieved by intermittent delivery of a VEGFR-TKI alternating with chemotherapy and sequential administration of VEGFR-TKI after front-line induction chemotherapy seem to be two reasonable therapeutic strategies to pursue; the latter is being investigated with sunitinib [39]. Third, not infrequently VEGFR-TKIs are highly toxic, which, in turn, might limit their success. In particular, several meta-analyses on the use of sorafenib and sunitinib to treat multiple solid malignancies concluded that these drugs are associated with increased risk of bleeding (relative risk, 2.0; $P=0.015$ ), arterial thromboembolism (relative risk, 3 . 03; $P=0.015$ ), and treatment-related mortality (relative risk, 2.23; $P=0.023$ ) [40-42]. In addition, VEGFR-TKIs may sometimes potentiate the side effects of chemotherapy, as is observed for cediranib. In fact, despite phase II interim results indicating greater ORR and PFS with cediranib $30 \mathrm{mg}$ daily given in conjunction with carboplatin-paclitaxel versus carboplatin-paclitaxel alone, the same VEGFRTKI plus chemotherapy combination was found to be associated with an excessive toxicity (which included gastrointestinal toxicity and febrile neutropenia) [29]. Unfortunately, when another phase II/III trial combined carboplatin-paclitaxel with a lower dose of cediranib given at $20 \mathrm{mg}$ daily no PFS improvement was observed [30]. These results emphasize the importance of adjustment of treatment on the basis of patients' tolerance, based mainly on implementation of early phase I/II testing in which pharmacokinetics and surrogative end-points could help find the right dosages and/or combination strategies.

Aflibercept, a recombinant fusion protein with sequence homology with native VEGFR-1 and VEGFR-2, is among other promising anti-angiogenic agents [43]. It acts as a soluble decoy receptor specifically designed to achieve pharmacological blockade of the VEGF pathway by high-affinity binding to the VEGF-A and $\mathrm{B}$ isoforms and the placental growth factor-1 and 2 isoforms [43]. Aflibercept achieved outstanding results in combination with chemotherapy for pretreated patients with metastatic colorectal cancer, in which it led to a significant prolongation of PFS $(\mathrm{HR}=0.75)$ and $\mathrm{OS}$ $(\mathrm{HR}=0.81)$ [44]. Unfortunately, similarly to VEGFR-TKIs, when added to chemotherapy (docetaxel) for pretreated advanced NSCLC patients, aflibercept was associated with a statistically significant improvement in ORR $(23.3 \%$ vs. 8 . $9 \%, P<.001)$ and PFS (5.2 months vs. 4.1 months, $\mathrm{HR}=0.82$; $P=0.0035)$ but not in the primary OS end-point $(\mathrm{HR}=1.01)$ [45]. Likewise, no better outcome was observed with the combination of chemotherapy with vascular disrupting agents, a category of drugs that target the endothelial cells of established tumor blood vessels and result in failure of the tumor vascular structure leading to blood and oxygen deprivation in the neoplastic tissue [46, 47]. Once again, these negative trials emphasize the importance of selection of patients by identification of biomarkers of sensitivity to anti-angiogenic treatment.

\section{Search for biomarkers of sensitivity to VEGFR-TKIs}

In some studies, as for vandetanib, several ancillary studies have been conducted with the purpose of correlating treatment outcome with putative biomarkers of sensitivity. In more detail, levels of $\mathrm{pVEGF}$ have been evaluated in an exploratory 
analysis of three randomized phase II trials in which the effect of vandetanib versus gefitinib, vandetanib versus either carboplatin or paclitaxel or the combination of both, and vandetanib (100 or $300 \mathrm{mg}$ daily) plus docetaxel versus docetaxel alone were evaluated [28, 48, 49]. In the first study, patients with low baseline pVEGF treated with vandetanib had a longer PFS than those treated with gefitinib (HR, 0.55; $95 \%$ CI, 0.35-0.86; $P=0.01$ ), with no difference for patients with high baseline pVEGF (HR, $1.0395 \% \mathrm{CI}, 0.60-1.75 ; P=$ 0.92; treatment-by-factor interaction test for VEGF, $P=0.08$ ) [50]. In the second study, PFS of patients with low baseline pVEGF receiving vandetanib was not significantly different from that of those receiving chemotherapy, although a trend in favor of the VEGFR-TKI was noted (HR, 0.80; $95 \% \mathrm{CI}, 0$. 41-1.56; $P=0.51$ ); conversely, PFS was shorter for patients with high baseline pVEGF who received vandetanib (HR, 1.6; $95 \% \mathrm{CI}, 0.81-3.15 ; P=0.17$; treatment-by-factor interaction test for VEGF, $P=0.09$ ) [50]. With regard to the combination treatment groups (vandetanib plus carboplatin-paclitaxel or vandetanib plus docetaxel) versus chemotherapy alone, low pVEGF levels were predictive of improved PFS only for vandetanib $100 \mathrm{mg}$ daily plus docetaxel PFS (HR, 0.25; $95 \%$ CI, $0.09-0.68 ; P=0.01$, treatment-by-factor interaction test for VEGF $P=0.14$ ); conversely, for patients with high pVEGF levels a trend toward higher PFS was observed for vandetanib plus carboplatin-paclitaxel (HR 0.47; $95 \%$ CI, $0.2-1.07 ; P=0.07$; treatment-by-factor interaction test $P=0.92$ ) [50]. Taken on the whole, these data suggest that low baseline pVEGF levels may be predictive of benefit from vandetanib monotherapy or vandetanib plus docetaxel. Also, patients with low baseline pVEGF may derive the same benefit from either vandetanib or carboplatin-paclitaxel, thus potentially favoring vandetanib with regard to improved tolerability.

A change during treatment in the pattern of expression of cytokines and angiogenic factors (CAFs) may provide important insights into the biological effects of treatment and identify drug-specific markers of activity and clinical benefit. Similarly to vandetanib monotherapy, an increase in pVEGF was associated with a higher risk of disease progression, whereas an increase in intracellular adhesion molecule 1 correlated with reduced risk [51].

Another study evaluated $31 \mathrm{CAFs}$ for NSCLCs treated with pazopanib [52]. Pazopanib induced significant changes in eight CAFs, of which changes in plasma soluble VEGFR-2 and IL-4 correlated significantly with tumor shrinkage.

\section{Conclusions}

Because angiogenesis is a very complex process, it is unlikely a single biomarker predicting treatment efficacy will be identified. For this reason, monitoring of different biomarkers at different times may enable more successful prediction of the effect of treatment. Alternative means of prediction are being investigated, in particular considering that biomarkers for antiangiogenic treatment may differ among cancer types. Interestingly, because VEGF blockade is believed to reduce tumor vascular permeability and perfusion, current research is also focusing on trying to correlate anti-angiogenic treatment with vascular responses by use of dynamic contrast-enhanced magnetic resonance imaging (DCE-MRI) rather than use of standard activity end-points, for example ORR, PFS, and OS [53]. Certainly, the only way to proceed consists in implementation of correlative clinical trials which may identify the most attractive biomarkers that, ultimately, will require prospective validation.

Conflict of interest Giulio Metro declares that he has no conflict of interest.

Matteo Cenci declares that he has no conflict of interest.

Alice Baldi declares that she has no conflict of interest.

Lucio Crinò declares that he has no conflict of interest.

\section{References}

Papers of particular interest, published recently, have been highlighted as:

- Of importance

1. Folkman J. Tumor angiogenesis: therapeutic implications. N Engl J Med. 1971;285:1182-6.

2. Pallis AG, Syrigos KN. Targeting tumor neovasculature in non-small-cell lung cancer. Crit Rev Oncol Hematol. 2012, In press.

3. - Jain RK, Carmeliet P. SnapShot: Tumor angiogenesis. Cell. 2012;149:1408-1408.e1. Synthetic description on how angiogenesis works and on the current status of anti-angiogenic therapy for solid malignancies.

4. Cohen MH, Gootenberg J, Keegan P, Pazdur R. FDA drug approval summary: bevacizumab (Avastin) plus Carboplatin and Paclitaxel as first-line treatment of advanced/metastatic recurrent nonsquamous non-small cell lung cancer. Oncologist. 2007; 12:713-8.

5. European Medicines Agency: Avastin. Available at http:// www.ema.europa.eu/ema/index.jsp?curl=pages/medicines/human/ medicines/000582/human_med_000663.jsp\&murl=menus/medicines/ medicines.jsp\&mid=WC0- 01 ac058001d125. Accessed January 2013.

6. Sandler A, Gray R, Perry MC, et al. Paclitaxel-carboplatin alone or with bevacizumab for non-small-cell lung cancer. N Engl J Med. 2006;355:2542-50.

7. Reck M, von Pawel J, Zatloukal P, et al. Phase III trial of cisplatin plus gemcitabine with either placebo or bevacizumab as first-line therapy for nonsquamous non-small-cell lung cancer: AVAil. J Clin Oncol. 2009;27:1227-34.

8. Reck M, von Pawel J, Zatloukal P, et al. Overall survival with cisplatin-gemcitabine and bevacizumab or placebo as first-line therapy for nonsquamous non-small-cell lung cancer: results from a randomised phase III trial (AVAiL). Ann Oncol. 2010;21:1804-49. 
9. - Soria JC, Mauguen A, Reck M, et al. Systematic review and metaanalysis of randomised, phase II/III trials adding bevacizumab to platinum-based chemotherapy as first-line treatment in patients with advanced non-small-cell lung cancer. Ann Oncol. 2013;24:20-30. Meta-analysis of randomized studies indicating that bevacizumab significantly prolongs PFS and OS when added to standard platinum-based chemotherapy as first-line therapy for patients with advanced non-squamous NSCLC.

10. National Comprehensive Cancer Network Guidelines. Available at http://www.nccn.org/professionals/physician_gls/ f_guidelines.asp\#nscl. Accessed January 2013.

11. Peters S, Adjei AA, Gridelli C, et al. Metastatic non-small-cell lung cancer (NSCLC): ESMO Clinical Practice Guidelines for diagnosis, treatment and follow-up. Ann Oncol. 2012;23 Suppl 7: vii56-64.

12. • Reck M, Barlesi F, Crinò L, et al. Predicting and managing the risk of pulmonary haemorrhage in patients with NSCLC treated with bevacizumab: a consensus report from a panel of experts. Ann Oncol. 2012;23:1111-20. Important review of the clinicopathological factors that predispose to the development of pulmonary haemorrhage while on bevacizumab therapy for advanced non-squamous NSCLC.

13. Goulart B, Ramsey S. A trial-based assessment of the cost-utility of bevacizumab and chemotherapy versus chemotherapy alone for advanced non-small cell lung cancer. Value Health. 2011;14:83645 .

14. Jayson GC, de Haas S, Delmar P, et al. Evaluation of plasma VEGF-A as a potential predictive pan-tumor biomarker for bevacizumab. [abstract 804]. Presented at the European Multidisciplinary Cancer Congress. Stockholm, Sweden; September 23-27, 2011.

15. Dowlati A, Gray R, Sandler AB, et al. Cell adhesion molecules, vascular endothelial growth factor, and basic fibroblast growth factor in patients with non-small cell lung cancer treated with chemotherapy with or without bevacizumab-an Eastern Cooperative Oncology Group Study. Clin Cancer Res. 2008;14:1407-12.

16. Hegde PS, Jubb AM, Chen D, et al. Predictive Impact of Circulating Vascular Endothelial Growth Factor in 4 Phase III Trials Evaluating Bevacizumab. Clin Cancer Res. 2013;19:92937.

17. Mok T, Gorbunova V, Juhasz E, et al. Biomarker analysis in BO21015 (ABIGAIL), a phase II randomised study of first-line bevacizumab (BEV) combined with carboplatin-gemcitabine (CG) or carboplatin-paclitaxel (CP) in patients (pts) with advanced or recurrent non-squamous non-small cell lung cancer (NSCLC). [abstract 9003]. Presented at the European Multidisciplinary Cancer Congress. Stockholm, Sweden; September 23-27, 2011.

18. Reck M, Gorbunova V, Juhasz E, et al. Tumor biomarker and plasma time course data from ABIGAIL, a phase II study of 1stline bevacizumab + chemotherapy in advanced non-squamous non-small-cell lung cancer (ns-NSCLC). [abstract 1236PD]. Presented at the 37th European Society for Medical Oncology Congress. Vienna, Austria; September 28-October 2, 2012.

19. Pallaud C, Reck M, Juhasz E, et al. Clinical genotyping and efficacy outcomes: exploratory biomarker data from the phase II ABIGAIL study of 1st-line bevacizumab + chemotherapy in nonsquamous non-small-cell lung cancer (ns-NSCLC). [abstract 1239P]. Presented at the 37th European Society for Medical Oncology Congress. Vienna, Austria; September 28-October 2, 2012.

20. Zhang W, Dahlberg SE, Yang D, et al. Genetic variants in angiogenesis pathway associated with clinical outcome in NSCLC patients (pts) treated with bevacizumab in combination with carboplatin and paclitaxel: Subset pharmacogenetic analysis of ECOG 4599. [abstract 8032]. Presented at the American Society of Clinical Oncology Congress. Orlando, USA; May 29-June 2, 2012.

21. Schneider BP, Wang M, Radovich M, et al. Association of vascular endothelial growth factor and vascular endothelial growth factor receptor-2 genetic polymorphisms with outcome in a trial of paclitaxel compared with paclitaxel plus bevacizumab in advanced breast cancer: ECOG 2100. J Clin Oncol. 2008;26:4672-8.

22. Lambrechts D, Claes B, Delmar P, et al. VEGF pathway genetic variants as biomarkers of treatment outcome with bevacizumab: an analysis of data from the AViTA and AVOREN randomised trials. Lancet Oncol. 2012;13:724-33.

23. Farhat FS, Tfayli A, Fakhruddin N, et al. Expression, prognostic and predictive impact of VEGF and bFGF in non-small cell lung cancer. Crit Rev Oncol Hematol. 2012;84:149-60.

24. Schneider BP, Gray R, Radovich M, et al. Prognostic and predictive value of tumor VEGF gene amplification in metastatic breast cancer treated with paclitaxel with and without bevacizumab; results from ECOG 2100 trial. Clin Cancer Res. 2013;19:1281-9.

25. Gatzemeier U, von Pawel J, Vynnychenko I, et al. First-cycle rash and survival in patients with advanced non-small-cell lung cancer receiving cetuximab in combination with first-line chemotherapy: a subgroup analysis of data from the FLEX phase 3 study. Lancet Oncol. 2011;12:30-7.

26. Dahlberg SE, Sandler AB, Brahmer JR, et al. Clinical course of advanced non-small-cell lung cancer patients experiencing hypertension during treatment with bevacizumab in combination with carboplatin and paclitaxel on ECOG 4599. J Clin Oncol. 2010;28:949-54.

27. Mir O, Coriat R, Cabanes L, et al. An observational study of bevacizumab-induced hypertension as a clinical biomarker of antitumor activity. Oncologist. 2011;16:1325-32.

28. Heymach JV, Paz-Ares L, De Braud F, et al. Randomized phase II study of vandetanib alone or with paclitaxel and carboplatin as first-line treatment for advanced non-small-cell lung cancer. J Clin Oncol. 2008;26:5407-15.

29. Goss GD, Arnold A, Shepherd FA, et al. Randomized, doubleblind trial of carboplatin and paclitaxel with either daily oral cediranib or placebo in advanced non-small-cell lung cancer: NCIC clinical trials group BR24 study. J Clin Oncol. 2010;28:49-55.

30. Laurie SA, Solomon BJ, Seymour L, et al. A randomized doubleblind trial of carboplatin plus paclitaxel (CP) with daily oral cediranib (CED), an inhibitor of vascular endothelial growth factor receptors, or placebo (PLA) in patients (pts) with previously untreated advanced non-small cell lung cancer (NSCLC): NCIC Clinical Trials Group study BR29. [abstract 7511]. Presented at the American Society of Clinical Oncology Congress. Chicago, USA; June 1-5, 2012.

31. Scagliotti G, Novello S, von Pawel J, et al. Phase III study of carboplatin and paclitaxel alone or with sorafenib in advanced nonsmall-cell lung cancer. J Clin Oncol. 2010;28:1835-42.

32. Paz-Ares LG, Biesma B, Heigener D, et al. Phase III, randomized, double-blind, placebo-controlled trial of gemcitabine/cisplatin alone or with sorafenib for the first-line treatment of advanced, nonsquamous non-small-cell lung cancer. J Clin Oncol. 2012;30:3084-92.

33. Scagliotti GV, Vynnychenko I, Park K, et al. International, randomized, placebo-controlled, double-blind phase III study of motesanib plus carboplatin/paclitaxel in patients with advanced nonsquamous non-small-cell lung cancer: MONET1. J Clin Oncol. 2012;30:2829-36.

34. Herbst RS, Sun Y, Eberhardt WE, et al. Vandetanib plus docetaxel versus docetaxel as second-line treatment for patients with advanced non-small-cell lung cancer (ZODIAC): a double-blind, randomised, phase 3 trial. Lancet Oncol. 2010;11:619-26. 
35. de Boer RH, Arrieta Ó, Yang $\mathrm{CH}$, et al. Vandetanib plus pemetrexed for the second-line treatment of advanced non-smallcell lung cancer: a randomized, double-blind phase III trial. J Clin Oncol. 2011;29:1067-74.

36. Metro G, Minotti V, Crinò L. Years of sorafenib investigation in advanced non-small cell lung cancer: is there a 'NExUS' linking an unsuccessful treatment and a potentially active one? J Thorac Dis. 2012;4(6):635-8.

37. Takezawa K, Okamoto I, Yonesaka K, et al. Sorafenib inhibits non-small cell lung cancer cell growth by targeting B-RAF in KRAS wild-type cells and C-RAF in KRAS mutant cells. Cancer Res. 2009;69:6515-21.

38. Pan F, Tian J, Zhang X, et al. Synergistic interaction between sunitinib and docetaxel is sequence dependent in human nonsmall lung cancer with EGFR TKIs-resistant mutation. J Cancer Res Clin Oncol. 2011;137:1397-408.

39. ClinicalTrials.gov Available at http://clinicaltrials.gov/ct/show/ NCT00693992. Accessed January 2013

40. Je Y, Schutz FA, Choueiri TK. Risk of bleeding with vascular endothelial growth factor receptor tyrosine-kinase inhibitors sunitinib and sorafenib: a systematic review and meta-analysis of clinical trials. Lancet Oncol. 2009;10:967-74.

41. Choueiri TK, Schutz FA, Je Y, et al. Risk of arterial thromboembolic events with sunitinib and sorafenib: a systematic review and meta-analysis of clinical trials. J Clin Oncol. 2010;28:2280-5.

42. Schutz FA, Je Y, Richards CJ, Choueiri TK. Meta-analysis of randomized controlled trials for the incidence and risk of treatment-related mortality in patients with cancer treated with vascular endothelial growth factor tyrosine kinase inhibitors. J Clin Oncol. 2012;30:871-7.

43. Stewart MW. Aflibercept (VEGF-TRAP): the next anti-VEGF drug. Inflamm Allergy Drug Targets. 2011;10:497-508.

44. Van Cutsem E, Tabernero J, Lakomy R, et al. Addition of aflibercept to fluorouracil, leucovorin, and irinotecan improves survival in a phase III randomized trial in patients with metastatic colorectal cancer previously treated with an oxaliplatin-based regimen. J Clin Oncol. 2012;30:3499-506.

45. Ramlau R, Gorbunova V, Ciuleanu TE, et al. Aflibercept and docetaxel versus docetaxel alone after platinum failure in patients with advanced or metastatic non-small-cell lung cancer: a randomized, controlled phase III trial. J Clin Oncol. 2012;30:3640-7.

46. Thorpe PE. Vascular targeting agents as cancer therapeutics. Clin Cancer Res. 2004;10:415-27.

47. Lara Jr PN, Douillard JY, Nakagawa K, et al. Randomized phase III placebo-controlled trial of carboplatin and paclitaxel with or without the vascular disrupting agent vadimezan (ASA404) in advanced non-small-cell lung cancer. J Clin Oncol. 2011;29:2965-71.

48. Natale RB, Bodkin D, Govindan R, et al. Vandetanib versus gefitinib in patients with advanced non-small-cell lung cancer: results from a two-part, double-blind, randomized phase ii study. J Clin Oncol. 2009;27:2523-9.

49. Heymach JV, Johnson BE, Prager D, et al. Randomized, placebocontrolled phase II study of vandetanib plus docetaxel in previously treated non small-cell lung cancer. J Clin Oncol. 2007;25:4270-7.

50. Hanrahan EO, Ryan AJ, Mann H, et al. Baseline vascular endothelial growth factor concentration as a potential predictive marker of benefit from vandetanib in non-small cell lung cancer. Clin Cancer Res. 2009;15:3600-9.

51. Hanrahan EO, Lin HY, Kim ES, et al. Distinct patterns of cytokine and angiogenic factor modulation and markers of benefit for vandetanib and/or chemotherapy in patients with non-small-cell lung cancer. J Clin Oncol. 2010;28:193-201.

52. Nikolinakos PG, Altorki N, Yankelevitz D, et al. Plasma cytokine and angiogenic factor profiling identifies markers associated with tumor shrinkage in early-stage non-small cell lung cancer patients treated with pazopanib. Cancer Res. 2010;70:2171-9.

53. O'Connor JP, Jackson A, Parker GJ, et al. Dynamic contrastenhanced MRI in clinical trials of antivascular therapies. Nat Rev Clin Oncol. 2012;9:167-77. 